\title{
Pérdidas de vidas, viviendas, infraestructura y embarcaciones por el tsunami del 27 de Febrero de 2010 en la costa central de Chile
}

\author{
Casualties, housing, infrastructure and vessel losses due to the February 27, 2010 Chile \\ tsunami on the central coast of Chile
}

\section{Manuel Contreras ${ }^{1}$ y Patricio Winckler ${ }^{2}$}

Fecha de entrega: 4 de junio 2013

Fecha de aceptación: 27 de agosto 2013

${ }^{1}$ Centro de Estudios Avanzados CEA y Facultad de Ingeniería, Universidad de Playa Ancha, Avda. Leopoldo Carvallo 270, Playa Ancha, Valparaíso, Chile, manuel.contreras@upla.cl

${ }^{2}$ Escuela de Ingeniería Civil Oceánica, Facultad de Ingeniería, Universidad de Valparaíso, Av. Borgoño 16344, Viña del Mar, Chile, patricio.winckler@uv.cl y School of Civil \& Environmental Engineering, Cornell University, 400 Triphammer Ap\#2, Ithaca, NY 14850, USA, paw87@cornell.edu

El terremoto $M w=8.8$ del 27 de febrero de 2010 en Chile se encuentra entre los sismos de mayor magnitud registrados por instrumentos a la fecha, posicionándose como el segundo más fuerte en la historia del país y uno de los diez más fuertes en el mundo registrados mediante instrumentos. Una de sus principales consecuencias fue la generación de un tsunami destructivo por la deformación del fondo marino. Las olas asolaron casi $600 \mathrm{~km}$ de la costa de Chile central y fueron la causa de un tercio de las víctimas fatales (181 sobre un total de 521), convirtiéndose asi en el tsunami con mayor número de muertes asociadas a este tipo de eventos en Sudamérica en los últimos 30 años. En el presente trabajo se reúnen y sistematizan una serie de antecedentes entre los que se contemplan informes, artículos y campañas de investigación y evaluación posttsunami, realizados en el litoral afectado. Se establece una caracterización cualitativa y estadística de las pérdidas de vidas humanas, viviendas, infraestructura costera y embarcaciones, separando pérdidas asociadas al tsunami de aquellas atribuibles exclusivamente al sismo. Mediante este catastro que integra información proveniente de fuentes de diferente naturaleza, se espera contribuir a evaluar los daños asociados al tsunami.

Palabras clave: evaluación de daños, terremoto y tsunami Chile 2010, estadistica de fallecidos
The $M w=8.82010$ Chile Earthquake is among the largest recorded by instruments to date, ranking as the second strongest in the country's history and one of the ten strongest worldwide. One of its main consequences was a destructive tsunami caused by the deformation of seafloor. The waves struck nearly 600 $\mathrm{km}$ of the coast of central Chile and the event caused about one third of the total casualties (181 out of 521), becoming the highest death toll due to this type of phenomena in South America in the last 30 years. In this paper the authors gather and systematize information from reports, papers and post-tsunami surveys carried out along the affected coastline. A qualitative and statistical characterization of the loss in lives, homes, infrastructure and fishing vessels is provided, separating tsunami effects to those attributable solely to the earthquake. Through the classification and integration of various data sources the authors expect to better explain and assess the damage specifically associated with the tsunami.

Keywords: damage assessment, 2010 Chile earthquake and tsunami, dead statistics

\section{Introducción}

Desde el punto de vista de los riesgos naturales, los terremotos de subducción con magnitudes de momento sísmico $M_{w}$ cercanas o superiores a 9.0, denominados mega terremotos por su capacidad destructiva, son los sismos más peligrosos (Madariaga, 1998). Este tipo de eventos ocurren frente a las costas de Chile producto del hundimiento de la densa placa de Nazca, bajo la menos 
densa Sudamericana (Cereceda et al., 2011), zona que concentra más del $25 \%$ de la energía liberada en forma sísmica en todo el mundo (Cisternas, 2011). Además de la enorme energía liberada, estos eventos son destructivos debido a su capacidad potencial de generar tsunamis (Levin y Nosov, 2009).

El 27 de febrero de 2010, hora local 3:34:08 AM (UTC - 3), la zona central de Chile fue sacudida por un mega terremoto de subducción que alcanzó una magnitud de momento sísmico $M_{w}=8.8$ (Vargas et al., 2011) y que fue capaz de generar un tsunami destructor. Este sismo afectó una zona poblada por cerca de trece millones de habitantes, más del 75\% de la población del país (CEPAL, 2010) y causó 521 víctimas fatales (Fritz et al., 2011). Producto de este desastre natural se perdieron 15000 puestos de trabajo (EERI, 2010), cerca del 3\% de la población chilena cayó bajo la línea de pobreza (Larrañaga y Herrera, 2010), cerca de 500000 viviendas quedaron con daños severos (HCDCh, 2011a,b) y más de 200000 familias perdieron sus hogares (Gobierno, 2010; MINVU, 2010). La magnitud alcanzada por el sismo lo posiciona como el segundo más fuerte en la historia del país y uno de los diez más fuertes registrados en el mundo mediante instrumentos sísmicos. Sólo es superado a nivel nacional por el terremoto de Valdivia de 1960, cuya intensidad de $M_{w}=9.5$ es la mayor registrada a nivel mundial hasta ahora mediante sismómetros (Kanamori, 1978; Benedetti, 2011). En términos económicos el terremoto produjo daños que se han estimado en treinta mil millones de dólares (Gobierno, 2010) equivalentes al 18\% del PIB nacional (OPS, 2010). Estos daños se descomponen en la pérdida de U\$10357 millones en infraestructura privada, U\$10538 millones en infraestructura pública y unos U\$9000 millones en bienes y servicios que se dejaron de producir por el terremoto (Gobierno, 2010). Aunque la última cifra se encuentra cuestionada (La Tercera, 2011), no hay duda que este desastre natural alcanzó los mayores costos para el país y toda Sudamérica desde 1900 a la fecha, llegando a ser el cuarto evento de origen sísmico más costoso a nivel mundial desde inicios del siglo XX (EM-DAT, 2012). Con respecto al número de afectados, que ascienden a 2.671.556 (EM-DAT, 2012), ocupa el lugar número once a nivel mundial y el segundo lugar en Sudamérica.

Diversos autores han realizado esfuerzos para evaluar los daños y efectos producidos por este sismo (CEPAL, 2010; GEER, 2010; EERI, 2010; Romero et al., 2010; Madariaga et al., 2010; Bresciani, 2010; Saragoni, 2011; CONAPACH, 2010; Larrañaga y Herrera, 2010; OPS, 2010; Yasuda et al., 2010; Martínez et al., 2011; Pulido et al., 2011; Fariña et al., 2012; Nahuelpan y Varas, 2010). Los daños fueron minimizados gracias a estrictas normas de construcción vigentes en Chile para la construcción de edificios (INN, 2003, 2009) e industrias (INN, 2003a). Estas normas han ido modificándose tras cada terremoto para aumentar los niveles de seguridad de las construcciones (Cisternas, 2011) y se buscó actualizarlas nuevamente para incorporar las lecciones de este sismo (Bitar, 2010; Betanzo, 2010). Con la aprobación de un reglamento que fija los requisitos de diseño y cálculo para el hormigón armado se busca la renovación de la norma para el diseño sísmico de edificios (Music et al., 2012).

Sin embargo, los reportes sobre daños que son atribuibles exclusivamente al tsunami son incompletos o se focalizan en aspectos puntuales del fenómeno. Martínez et al. (2011) y Morales (2010) se restringen a evaluar los daños sólo a la zona sur afectada. Winckler et al. (2010) analiza los efectos sobre el poblado de San Juan Bautista, en el Archipiélago de Juan Fernández. Marín et al. (2010) considera los efectos en la pesca artesanal, restringiéndose a tres de las cuatro regiones afectadas y a nivel agregado. Quezada et al. (2010, 2010a, 2012), Fritz et al. (2011), Vingy et al. (2011), Yasuda et al. (2010), Jaramillo et al. (2012) y González et al. (2012) consideran aspectos morfológicos e hidrodinámicos sin detenerse en evaluar daños. Fariña et al. (2010), Urbina (2011), Rubio y Basic (2011), Lazcano y Retamal (2011) y Valdovinos et al. (2012) identifican algunos efectos ambientales. Winckler et al. (2011) identifican diversos tipos de daños, sin efectuar una cuantificación. Olsen et al. (2012) y Robertson et al. (2012) analizan los daños por el tsunami en edificios e infraestructura; las instalaciones industriales son abordadas por Zareian et al. (2012); mientras los daños en puertos son estudiados por Zareian et al. (2012), Brunet et al. (2012) y Bray et al. (2012). Robertson et al. (2012) analiza los efectos sobre líneas de vida, reconstrucción y recuperación. Por último Aránguiz (2010) entre otros autores, procura reconstruir por medio de modelaciones numéricas el fenómeno. 
El presente trabajo busca contribuir al conocimiento y evaluación de los daños y costos específicos del tsunami a lo largo de la costa de Chile, en lo relativo a pérdidas de vidas, viviendas, infraestructura y embarcaciones. Los resultados surgen de la integración de fuentes de información de diferente naturaleza y son presentados en forma desagregada, con el objetivo de complementar información existente. Este tipo de antecedentes pueden resultar útiles para identificar las vulnerabilidades del litoral y planificar de mejor forma el uso de la zona costera y las medidas de contingencia ante este tipo de riesgos naturales.

\section{Metodología}

Entre el 1 de marzo y el 23 de mayo de 2010, se efectuó un levantamiento de la zona afectada, recorriéndose un total de $800 \mathrm{~km}$ de la costa, en el marco del Post Tsunami Survey UNESCO - ITIC, Chile 2010 (Fritz et al., 2011). Se utilizó la metodología UNESCO-IOC (1998) para realizar entrevistas a testigos en la zona afectada, junto con registrar las marcas físicas dejadas por las ondas del tsunami, entre las que destacan la intrusión horizontal, la profundidad y cota de inundación (Figura 1). La información obtenida de este levantamiento constituye el punto de partida de este trabajo. En la Figura 2 se muestran las localidades con mayor población afectadas por el tsunami que se mencionan en el texto.

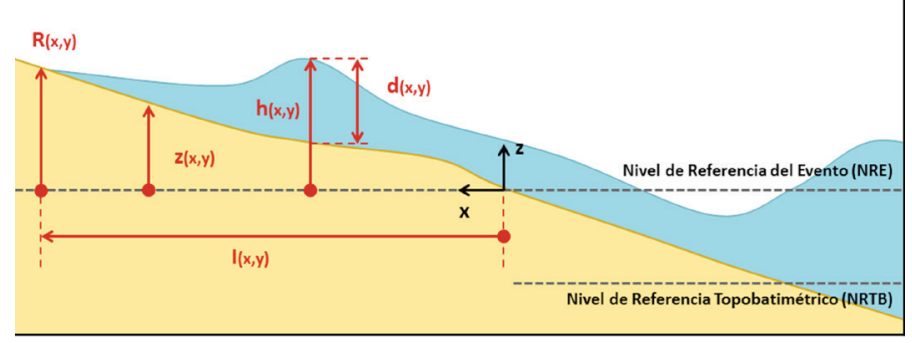

Figura 1: Nomenclatura utilizada para referir a los diferentes planos verticales e intrusión horizontal de inundación por tsunami, con respecto a un cierto nivel de referencia del evento (NRE) y el de referencia topo-batimétrico (NRTB). R: runup o máxima cota de inundación, $\mathrm{z}$ : cota de terreno, h: cota de inundación, d: profundidad de inundación e I: máxima intrusión horizontal. Estas variables dependen de las coordenadas horizontales (x, y).

En forma complementaria, se analizaron antecedentes disponibles en la prensa escrita, informes de equipos de estudio especializados (GEER, 2010) y antecedentes de la investigación post tsunami reunidos por la Fiscalía Nacional de Chile (Fiscalía, 2011).

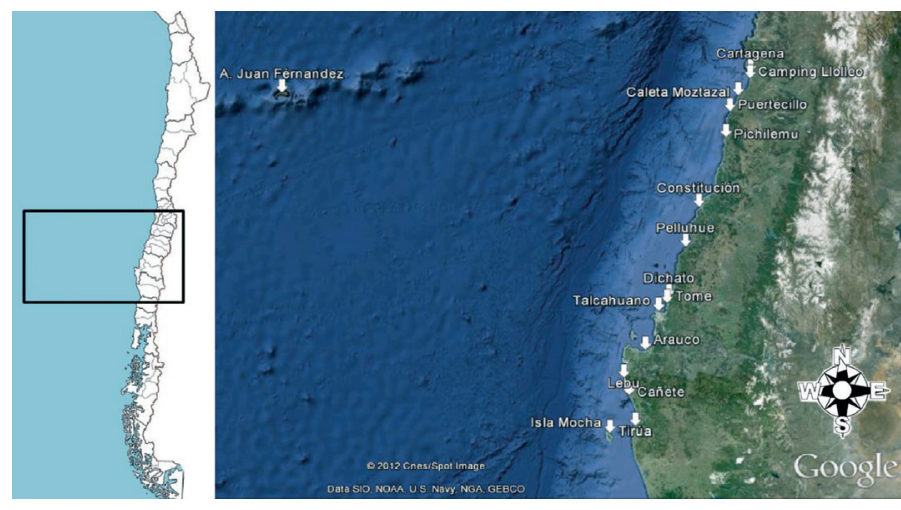

Figura 2: Zona afectada por el tsunami en Chile central. Se señalan localidades con mayor población estudiadas en el presente trabajo. Adaptado de Google Earth

Los antecedentes fueron organizados de acuerdo a su localización geográfica, y clasificados bajo las siguientes categorías:

- Pérdidas en vidas humanas

- Pérdidas en viviendas livianas

- Pérdidas en infraestructura

- Pérdidas en embarcaciones menores

Para completar los antecedentes se realizó un estudio exploratorio de referencias bibliográficas, considerando sólo documentos que incluían datos y estadísticas con indicación de fuente, informes oficiales, y/o que correspondían a publicaciones o reportes técnicos con revisión editorial. Del sistema Redatam ${ }^{+}$(INE, 2013) se extrajo el número de habitantes en entidades poblacionales que fueron afectadas por el tsunami.

\section{Pérdidas en vidas humanas}

El tsunami de 2010 representa el evento Sudamericano con mayor número de víctimas desde el ocurrido en Tumaco en las costas de Colombia en el año 1979 (Fritz et al., 2011), el cual cobró la vida de más de 220 residentes de San Juan (Herd et al., 1981), convirtiéndose así en el tsunami con mayor número de muertes asociadas a este tipo de eventos en Sudamérica en los últimos 30 años. Según las cifras oficiales del Ministerio del Interior y la investigación llevada a cabo por la Fiscalía Nacional de Chile (Fiscalía, 2011), el tsunami cobró 181 vidas, de las cuales 156 fueron identificadas, mientras que 25 se encontraban 
desaparecidas a mayo de 2013. Esta cifra es mayor que las 124 víctimas reportadas por Fritz et al. (2011), lo que se atribuye a la diferente naturaleza de ambos estudios: el primero orientado a definir responsabilidades y el segundo con un enfoque en la física del fenómeno. El listado incluido en el informe de la Fiscalía indica que el 53\% de las víctimas fueron varones, y las edades fluctuaron entre los 7 meses hasta los 95 años. El 50\% de los fallecidos y desaparecidos eran mayores de 50 años, mientras que el $21 \%$ eran niños y jóvenes menores de 18 años. En la Tabla 1 se muestra el número de víctimas clasificadas por localización geográfica, contrastado con los registros de runup y máxima intrusión horizontal (Fritz et al., 2011).

La correlación entre el porcentaje de víctimas con respecto a la población de cada localidad y el runup, y el porcentaje de víctimas con respecto a la población de cada localidad versus la intrusión horizontal se ilustran para cada localidad en la Tabla 1. En la Figura 3 se observa una correlación entre el runup y el porcentaje de víctimas por localidad de $\mathrm{r}^{2}=0.64$. Asimismo, existe una muy baja correlación entre la intrusión horizontal y el porcentaje de víctimas por localidad $\left(\mathrm{r}^{2}=0.031\right)$, lo que indica que el runup es mejor predictor del número de víctimas. Los bajos valores de correlación, no obstante, pueden explicarse por el reducido número de localidades afectadas $(\mathrm{n}=16)$. Estos resultados son válidos sólo para el evento de 2010 pero pueden ser contrastados con otros eventos recientes, habida cuenta de la gran cantidad de datos generados en los últimos posttsunami surveys (Synolakis y Okal, 2005).

Gran parte de las víctimas no tenía domicilio en las zonas costeras, sino que eran turistas provenientes de las ciudades interiores de Talca, Santiago y Curicó. Por ejemplo, se sabe que de las 48 víctimas por el tsunami en la localidad de Pelluhue (Tabla 1), 41 eran turistas (OPS, 2010). Sólo doce de los fallecidos por el tsunami eran pescadores artesanales, de una población que supera los 75000 (Marin et al., 2010), y la mayoría fue víctima de las ondas a bordo de sus botes rescatando gente que era tragada por el mar la madrugada del maremoto (HCDCh, 2011). Se debe observar que Marin et al. (2010), consigna que el número de pescadores artesanales fallecidos por el tsunami son 8 , lo que se debe a que en su estudio no se analizó lo ocurrido en la $\mathrm{V}$ región.
Tabla 1: Localización geográfica de número de víctimas, incluyendo desaparecidos, y máximos runup e intrusión de la onda de tsunami en cada localidad.

\begin{tabular}{|c|c|c|c|c|c|c|c|c|c|}
\hline 莺 & 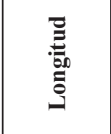 & 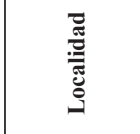 & Ü & 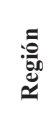 & $\begin{array}{l}\Xi \\
\hat{E} \\
\hat{E} \\
\hat{E}\end{array}$ & 总 & 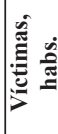 & 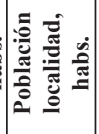 & \\
\hline $33^{\circ} 39^{\prime} \mathrm{S}$ & $78^{\circ} 51^{\prime} \mathrm{W}$ & $\begin{array}{c}\text { Juan } \\
\text { Fernández }\end{array}$ & $\begin{array}{c}\text { Juan } \\
\text { Fernández }\end{array}$ & \multirow{4}{*}{ V } & 18 & 295 & 16 & 885 & 1.81 \\
\hline $33^{\circ} 31^{\prime} \mathrm{S}$ & $71^{\circ} 37^{\prime} \mathrm{W}$ & Cartagena & Cartagena & & 4 & 103 & 1 & 15302 & 0.01 \\
\hline $33^{\circ} 36^{\prime} \mathrm{S}$ & $71^{\circ} 37.5^{\prime} \mathrm{W}$ & $\begin{array}{l}\text { Camping } \\
\text { Llolleo }\end{array}$ & San Antonio & & 4 & 455 & 5 & 12488 & | 0.04 \\
\hline $33^{\circ} 50^{\prime} \mathrm{S}$ & $71^{\circ} 49^{\prime} \mathrm{W}$ & $\begin{array}{c}\text { Caleta } \\
\text { Moztazal }\end{array}$ & $\begin{array}{c}\text { Santo } \\
\text { Domingo }\end{array}$ & & 14 & 434 & 1 & 79 & 1.27 \\
\hline $34^{\circ} 03^{\prime} \mathrm{S}$ & $71^{\circ} 57^{\prime} \mathrm{W}$ & Puertecillo & Navidad & \multirow{2}{*}{ VI } & 12 & $\begin{array}{c}\text { Sin } \\
\text { dato }\end{array}$ & 2 & 184 & 1.09 \\
\hline $34^{\circ} 24^{\prime} \mathrm{S}$ & $72^{\circ} 01^{\prime} \mathrm{W}$ & Pichilemu & Pichilemu & & 6 & 270 & 3 & 2178 & 0.14 \\
\hline $35^{\circ} 20^{\prime} \mathrm{S}$ & $72^{\circ} 27^{\prime} \mathrm{W}$ & Constitución & Constitución & \multirow{2}{*}{ VII } & 29 & 643 & 55 & 4167 & 1.32 \\
\hline $35^{\circ} 52^{\prime} \mathrm{S}$ & $72^{\circ} 40^{\prime} \mathrm{W}$ & Pelluhue & Pelluhue & & 14 & 682 & 48 & 4596 & 1.04 \\
\hline $36^{\circ} 32^{\prime} \mathrm{S}$ & $72^{\circ} 56^{\prime} \mathrm{W}$ & Dichato & Tomé & \multirow{8}{*}{ VIII } & 11 & 856 & 12 & 3488 & 0.34 \\
\hline $36^{\circ} 36.7 \mathrm{~S}$ & $72^{\circ} 58^{\prime} \mathrm{W}$ & $\begin{array}{c}\text { Caleta Los } \\
\text { Bagres }\end{array}$ & Tomé & & 8 & 130 & 1 & 2178 & 0.05 \\
\hline $36^{\circ} 43^{\prime} \mathrm{S}$ & $73^{\circ} 06^{\prime} \mathrm{W}$ & Talcahuano & Talcahuano & & 7 & 340 & 21 & 81000 & 0.03 \\
\hline $37^{\circ} 14^{\prime} \mathrm{S}$ & $73^{\circ} 19^{\prime} \mathrm{W}$ & Arauco & Arauco & & 4 & 123 & 1 & 16204 & 0.01 \\
\hline $37^{\circ} 36^{\prime} \mathrm{S}$ & $73^{\circ} 40^{\prime} \mathrm{W}$ & Costa Mina & Lebu & & 13 & 144 & 4 & 450 & 0.89 \\
\hline $37^{\circ} 50^{\prime} \mathrm{S}$ & $73^{\circ} 35^{\prime} \mathrm{W}$ & Cañete & Cañete & & 9 & 573 & 5 & 17797 & 0.03 \\
\hline $38^{\circ} 14.7^{\prime} \mathrm{S}$ & $73^{\circ} 29^{\prime} \mathrm{W}$ & Quidico & Tirúa & & 8 & 729 & 2 & 1013 & 0.20 \\
\hline $38^{\circ} 20^{\prime} \mathrm{S}$ & $73^{\circ} 54^{\prime} \mathrm{W}$ & Isla Mocha & Lebu & & 18 & 582 & 4 & 584 & 0.68 \\
\hline & & & & & & Total & 181 & 162593 & 0.11 \\
\hline
\end{tabular}

a)

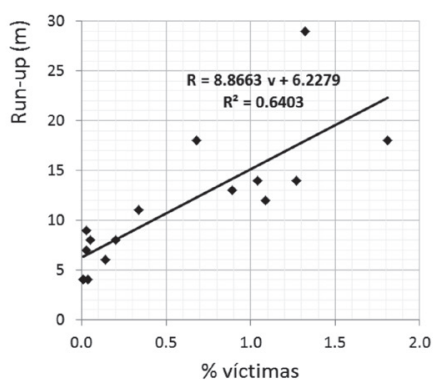

b)

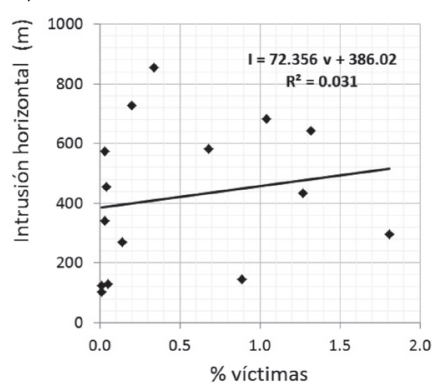

Figura 3: a) Runup versus \% de víctimas y b) intrusión horizontal versus \% de víctimas, datos adaptados de Fritz et al. (2011), Fiscalía (2011) e INE (2013).

\section{Pérdidas en viviendas livianas}

El comportamiento de las viviendas livianas ante el tsunami de 2010 ha sido escasamente documentado en la literatura científica. Los estudios se focalizan sólo en los efectos del sismo sobre viviendas de albañilería (Astroza et al., 2012) 
y adobe (D'Ayala et al., 2012), o en el comportamiento del hormigón armado en edificios altos (Westenenk et al., 2012; Betanzo, 2010). Winckler et al. (2010) analizan en forma somera el efecto de las cargas hidrodinámicas del tsunami en el poblado de San Juan Bautista, Archipiélago Juan Fernández, donde detectaron fallas por impacto y flotación en prácticamente todas las edificaciones ubicadas en la zona inundada. A objeto de complementar la escasa documentación disponible, se efectúa: i) un análisis cualitativo del tipo de fallas de viviendas livianas en Iloca, y ii) un catastro de viviendas destruidas por localidad (Tabla 2).

En el marco del levantamiento post tsunami (Fritz et al., 2011), se efectuaron mediciones y registraron los efectos del tsunami en Iloca sur (Figura 4a a 4d). La Figura 4e ilustra un perfil del sector afectado, con una duna de cota cercana a $+3.5 \mathrm{~m}$ en la zona alta de la playa, y una planicie interior donde se encontraban las edificaciones. En el perfil se registró una máxima intrusión horizontal de $250 \mathrm{~m}$, una profundidad máxima de escurrimiento de $1.2 \mathrm{~m}$ y un runup de $3 \mathrm{~m}$. Estos valores son comparables en orden de magnitud a otras mediciones cercanas, por lo que el perfil puede considerarse como típico para la localidad.

El efecto sobre las viviendas fue variable, siendo en general de fallo total en instalaciones de madera, parcial en aquellas de adobe o albañilería y menor en aquellas de hormigón armado. En la Figura 5a se ilustran las tres tipologías ubicadas en las proximidades del perfil de la Figura 4e. La vivienda de madera (izquierda) experimentó una pérdida total, en tanto que la de albañilería (centro) sufrió daños menores en vidrios y terminaciones. La vivienda de adobe (derecha), sufrió daños moderados, pero diez días después del tsunami, aún mostraba sus paredes húmedas por la absorción del agua. El efecto de la humedad en las propiedades mecánicas del material, si lo hubo, no fue cuantificado debido a la naturaleza del levantamiento, donde se privilegia la cobertura rápida de grandes zonas geográficas por sobre el análisis detallado de estructuras específicas.

En general, las viviendas de madera tipo "palafito" no experimentaron daños significativos por ubicarse a una cierta altura del suelo. La solución de elevar con pilares la construcción (Figura 5b) resultó por lo general exitosa, salvo en aquellos casos en que la inundación llegó a nivel del piso de la casa, o cuando ésta sólo se encontraba apoyada por gravedad, sin sistemas de anclaje.

La inundación provocó la flotación de aquellas construcciones livianas que no estaban firmemente ligadas a las fundaciones y en ocasiones, fueron arrastradas por el flujo. En la Figura 5c se muestran las cadenas de una vivienda de albañilería reforzada, encontradas a decenas de metros de su posición original. En la Figura 5d se ilustran los restos del segundo piso de una vivienda de madera desplazada desde su posición original, cuyo primer piso colapsó, y una segunda vivienda con el primer piso de hormigón armado en construcción sin daños en la misma localidad.
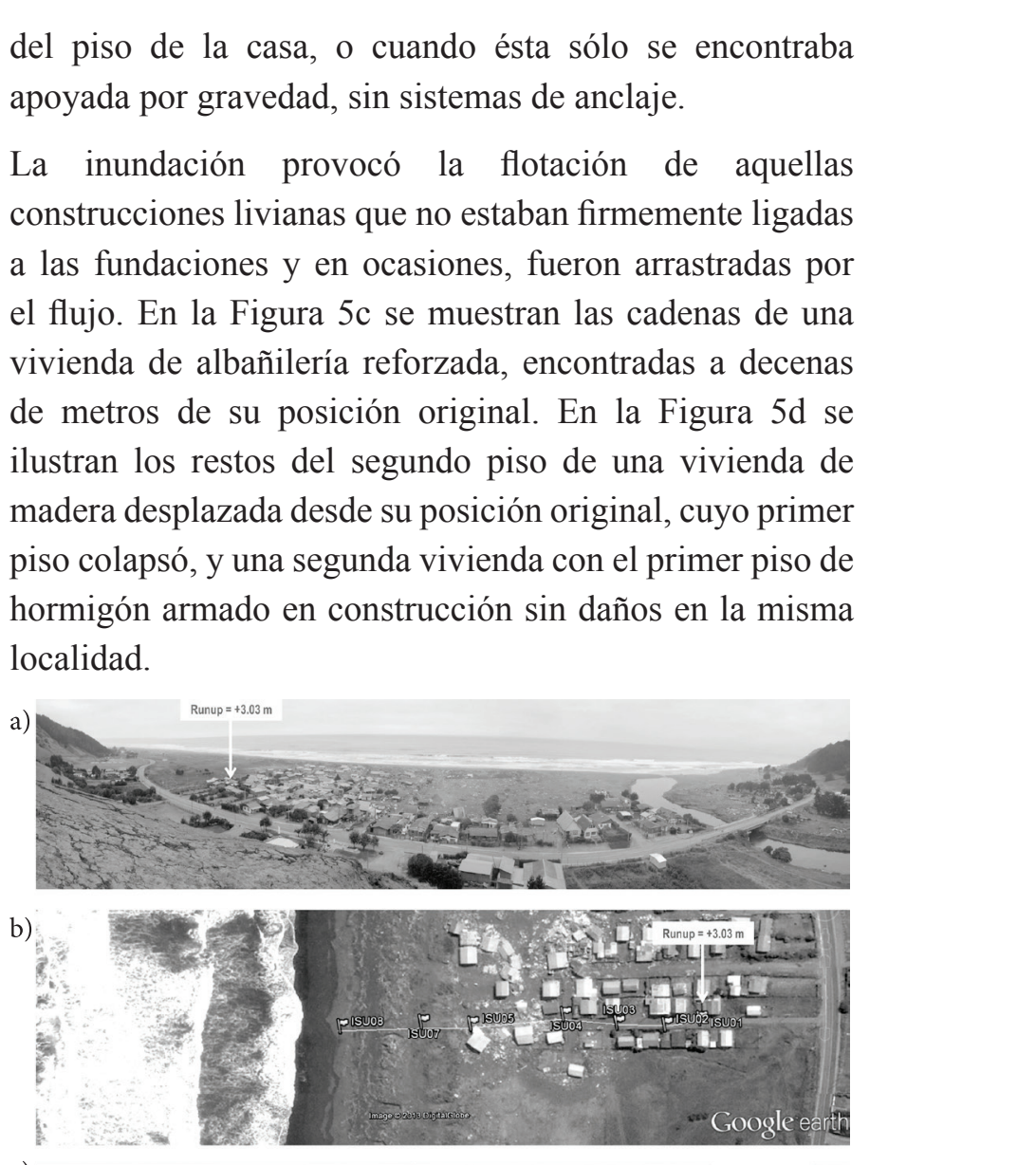

c)

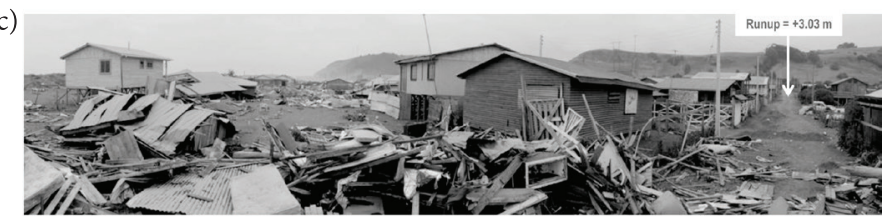

d)
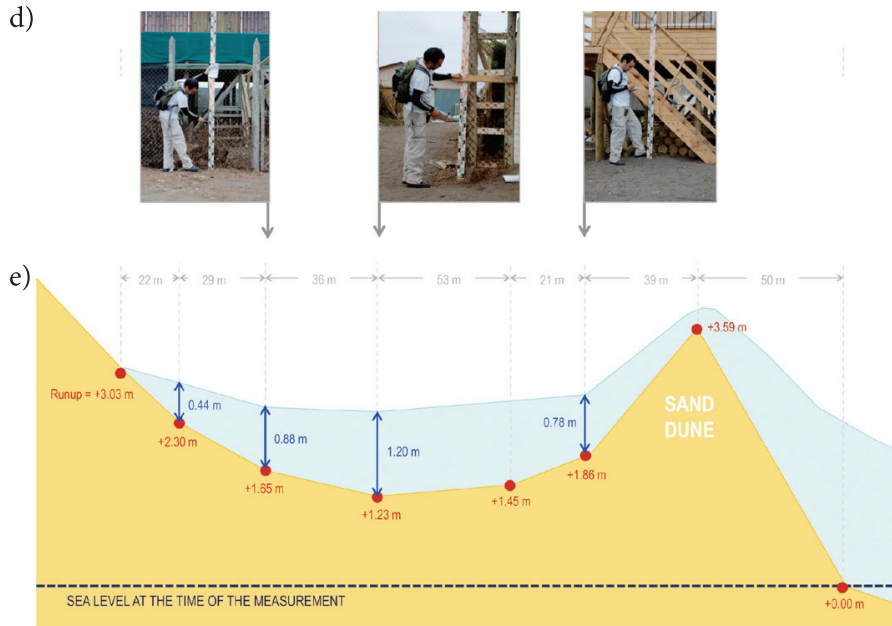

Figura 4: a) Vista general de la localidad de Iloca (345ㅇ'S). Se señala el punto donde se pudo determinar runup, b) vista de planta del perfil realizado para determinar el runup, c) vista desde la dirección donde ingresó la onda de tsunami, al fondo se identifica el punto de runup, d) registros de profundidades de inundación y e) corte vertical de la inundación. 
a)

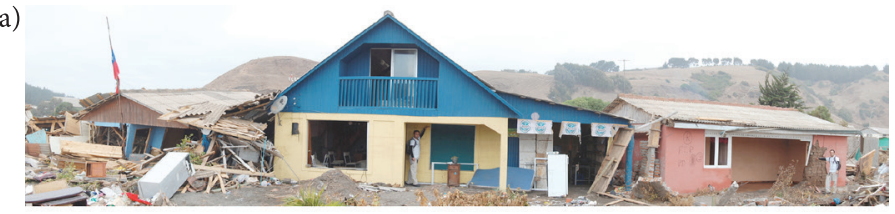

b)

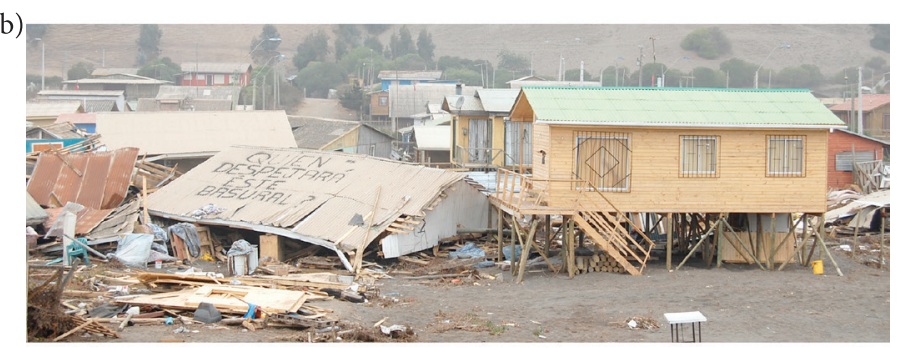

c)

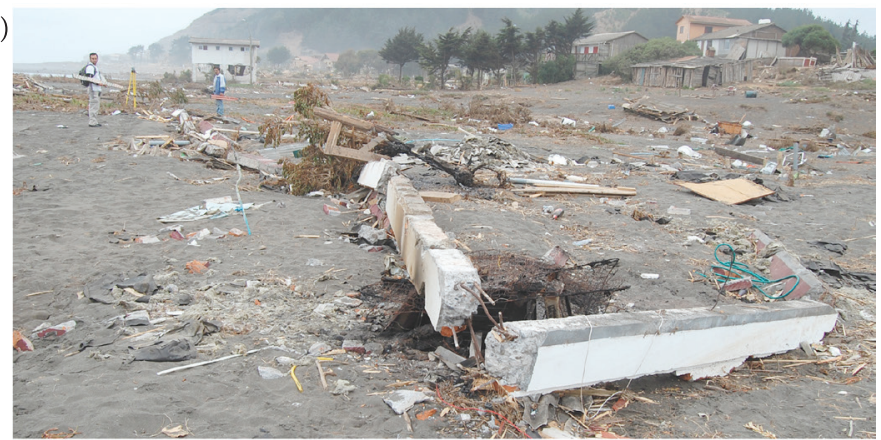

d)

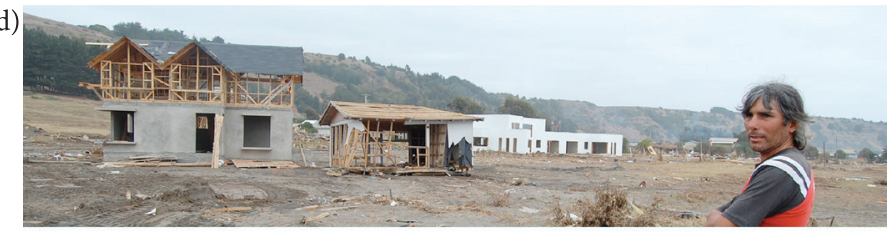

Figura 5: Diferentes tipos de fallas en Iloca ( $34^{\circ} 56^{\prime}$ 'S). a) Fallas en diferentes tipologías estructurales, b) falla de tipologías estructurales y éxito de viviendas de madera elevadas con pilares y un sistema de anclaje, c) cadenas de vivienda encontradas a decenas de metros de su posición original (al fondo), d) restos del segundo piso de una vivienda de madera desplazada desde su posición original y vivienda con primer piso en construcción. En primer plano, maestro constructor de la casa sin daño.

En algunos casos, la fuerza del impacto de las ondas y la cota de inundación fue de tal magnitud que incluso destruyó viviendas de hormigón armado, como el caso de una casa de tres pisos ubicada en la ribera del río Maule, a $2.5 \mathrm{~km}$ de la desembocadura (Figura 6). La vivienda, constituida por columnas, vigas y bloques de hormigón, experimentó pérdida parcial de la techumbre, ubicada a unos $12 \mathrm{~m}$ sobre el nivel del mar.

Otro ejemplo lo constituye Llolleo, V región, donde se observaron daños en fundaciones de viviendas livianas que permitieron la flotación de las mismas y fallos en fundaciones de estructuras mayores (Figura 7). Los "poyos" (Figura 7a) construidos con base de hormigón

de unos 20 a $30 \mathrm{~cm}$, colapsaron debido a la socavación general del suelo y a la socavación local producto de la interacción del flujo con la estructura. La Figura $7 \mathrm{~b}$ ilustra la socavación local en una cámara de hormigón armado. La Figura 7c muestra viviendas completamente destruidas debidas a las fuerzas hidrodinámicas de presión, arrastre y flotación.

En la Tabla 2 se resume la información recabada de diversas fuentes sobre el número de viviendas dañadas exclusivamente por el tsunami por localidad. La cifra total alcanza 17392.

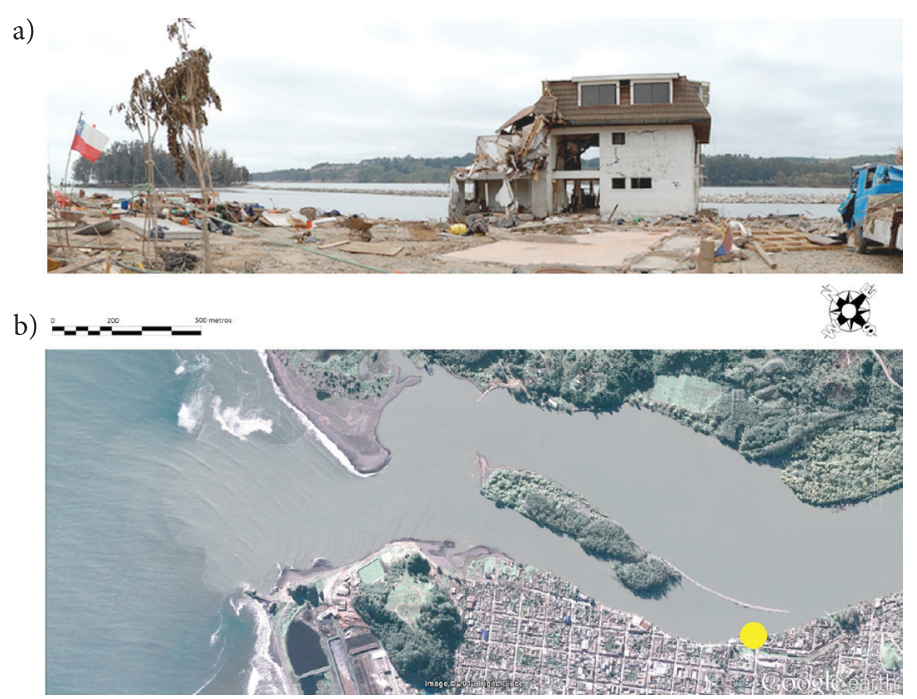

Figura 6: a) Falla por el impacto del tsunami en casa de hormigón armado ubicada en la ribera del Río Maule (35²0'S) y b) localización indicada en puntero amarillo.

a)

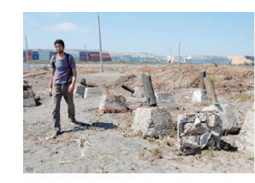

d)

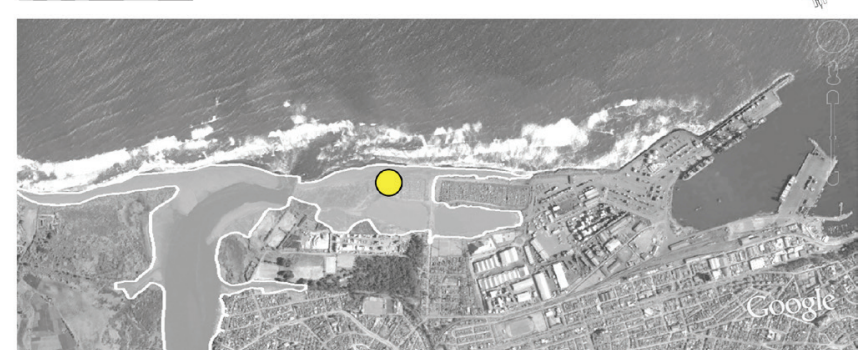

Figura 7: Falla de viviendas livianas en Llolleo (333ㅇ'S). a) y c) fundaciones donde reposaban las viviendas de material ligero que fueron arrastradas por el tsunami, éstas fallaron porque la vivienda no estaba anclada a los pilotes sino con clavos y pernos menores, b) socavación en torno a una estructura de hormigón y d) localización indicada en círculo amarillo. 
Tabla 2: Número de fallecidos por localidad donde se comprobó la existencia de viviendas dañadas severamente por el tsunami.

\begin{tabular}{|c|c|c|c|c|c|c|}
\hline Localidad & Comuna & Región & Fallecidos & $\begin{array}{c}\text { Área } \\
\text { inundada, } \\
\mathrm{km}^{2}\end{array}$ & $\begin{array}{l}\text { Viviendas } \\
\text { dañadas }\end{array}$ & Fuente \\
\hline Llolleo & San Antonio & \multirow{3}{*}{ V } & 5 & 0.43 & 168 & IMSA 2010; Contreras et al. 2012 \\
\hline Moztazal & Santo Domingo & & 1 & S.I. & 96 & INE 2010 \\
\hline Isla Robinson Crusoe & Juan Fernández & & 16 & 0.58 & 160 & Winckler et al. 2010, INE 2010 \\
\hline Matanzas & Navidad & \multirow{6}{*}{ VI } & - & S.I. & 2 & CONAPACH 2010 \\
\hline Puertecillo & Litueche & & 2 & S.I. & 56 & INE 2010 \\
\hline Tanumé & \multirow{3}{*}{ Pichilemu } & & 2 & S.I. & 51 & INE 2010 \\
\hline Cahuil & & & - & S.I. & 6 & CONAPACH 2010 \\
\hline Bucalemu & & & - & S.I. & 5 & CONAPACH 2010 \\
\hline Paredones & Paredones & & - & S.I. & 6 & INE 2010 \\
\hline Boyeruca & Vichuquen & \multirow{15}{*}{ VII } & - & S.I. & 29 & CONAPACH 2010 \\
\hline Licanten & \multirow{3}{*}{ Licanten } & & - & S.I. & 43 & INE 2010 \\
\hline Duao & & & - & S.I. & 27 & CONAPACH 2010 \\
\hline Iloca & & & - & S.I. & 391 & INE 2010 \\
\hline Curepto & Curepto & & - & S.I. & 6 & INE 2010 \\
\hline Constitución & \multirow{4}{*}{ Constitución } & & - & 2.43 & 2898 & INE 2010 \\
\hline Río Maule & & & 2 & S.I. & 130 & CONAPACH 2010 \\
\hline Pellines & & & - & S.I. & 6 & CONAPACH 2010 \\
\hline Loanco & & & - & S.I. & 22 & CONAPACH 2010 \\
\hline Chanco & Chanco & & - & S.I. & 10 & CONAPACH 2010 \\
\hline Caleta Blanca & \multirow{5}{*}{ Pelluhue } & & 3 & S.I. & 38 & CONAPACH 2010 \\
\hline El Mariscadero & & & 3 & S.I. & 9 & CONAPACH 2010 \\
\hline Centro Pelluhue & & & 1 & S.I. & 41 & CONAPACH 2010 \\
\hline Cardenal & & & - & S.I. & 10 & CONAPACH 2010 \\
\hline Tregualemu & & & - & S.I. & 46 & INE 2010 \\
\hline Cobquecura & \multirow{2}{*}{ Cobquecura } & \multirow{30}{*}{ VIII } & - & 0.00 & - & GORE 2010 \\
\hline Rinconada de Taucu & & & - & S.I. & 179 & INE 2010; Morton et al., 2010 \\
\hline Treguaco & Treguaco & & - & S.I. & 84 & INE 2010; Morales 2010 \\
\hline Perales & Coelemu & & - & 0.57 & 95 & GORE 2010 \\
\hline Purema & \multirow{8}{*}{ Tomé } & & - & 0.25 & S.I. & Morton et al., 2010 \\
\hline Dichato & & & 12 & 0.85 & 1343 & GORE 2010; Martínez et al. 2011 \\
\hline Pingueral & & & - & S.I. & 25 & GEER 2010; Morales 2010 \\
\hline Coliumo & & & - & 0.45 & 114 & GORE 2010 \\
\hline Caleta del Medio & & & - & 0.05 & 52 & GORE 2010 \\
\hline Los Morros & & & - & 0.04 & 45 & GORE 2010 \\
\hline Tomé & & & - & 0.10 & 143 & INE 2010 \\
\hline Caleta Los Bagres & & & 1 & S.I. & 111 & INE 2010 \\
\hline Penco & Penco & & - & 0.85 & 326 & GORE 2010 \\
\hline Caleta Tumbes & \multirow{6}{*}{ Talcahuano } & & 2 & 0.05 & 40 & GORE 2010 \\
\hline Caleta Puerto Inglés & & & 1 & S.I. & 117 & INE 2010; Morales 2010 \\
\hline Caleta Cantera & & & 1 & S.I. & 14 & INE 2010; Morales 2010 \\
\hline Centro de Talcahuano & & & 3 & 11.04 & 7391 & INE 2010 \\
\hline Ruta Interportuaria & & & 3 & S.I. & 2 & INE 2010 \\
\hline Lenga & & & - & S.I. & S.I. & Morales 2010 \\
\hline Lota & Lota & & - & 0.36 & 803 & INE 2010 \\
\hline Pto. Sur, I. Sta. María & Coronel & & - & 0.00 & - & GORE 2010 \\
\hline Laraquete & \multirow{4}{*}{ Arauco } & & - & 0.20 & 198 & INE 2010 \\
\hline Raqui - Las Peñas & & & - & S.I. & S.I. & Morales 2010 \\
\hline Tubul & & & - & 0.75 & 700 & Morales 2010 \\
\hline Llico & & & 1 & 3.59 & 320 & Morales 2010; Martínez et al. 2011 \\
\hline Costa Mina & Lebu & & 4 & 1.69 & 819 & INE 2010; Martínez et al. 2011 \\
\hline Quidico & \multirow{2}{*}{ Tirúa } & & 2 & 0.08 & 30 & GORE 2010 \\
\hline Tirúa & & & - & 0.80 & 145 & Morales 2010 \\
\hline Isla Mocha & \multirow[t]{2}{*}{ Lebu } & & 4 & 2.81 & 40 & GORE 2010; La Tercera 12/5/12 \\
\hline & & & $69(*)$ & 27.72 & 17392 & \\
\hline
\end{tabular}

S.I.: Sin información $(*)$ se refiere al total de fallecidos en lugares donde hubo infraestructura dañada. Una parte importante de los fallecidos se encontraban acampando en lugares rurales sin vivienda, como es el caso de isla Orrego, esto explica que solo aparezca en esta tabla el $38 \%$ de los fallecidos. 


\section{Pérdidas en infraestructura}

En Talcahuano, la Base Naval, los astilleros y maestranzas de la Armada de Chile ASMAR y el Edificio Consistorial sufrieron importantes daños, al igual que la infraestructura portuaria, afectando un gran número de contenedores que fueron barridos por la onda de tsunami (OPS, 2010; Olsen et al., 2011; Brunet et al., 2011).

Una situación similar se registró en el Puerto de San Antonio (IMSA, 2010). En la Tabla 3 se muestra el resumen del número de infraestructuras costeras de uso público (41) afectadas por el tsunami, por tipo y región, obtenida a partir de información gubernamental (Gobierno, 2010). Resalta que además de las cuatro regiones principalmente afectadas por el sismo (Valparaíso, O’Higgins, Maule y Bío Bío), en las regiones de la Araucanía y los Ríos también se reportaron daños producto del tsunami en instalaciones de pescadores artesanales y defensas costeras. Se puede considerar entonces que el tsunami afectó a seis regiones del país.

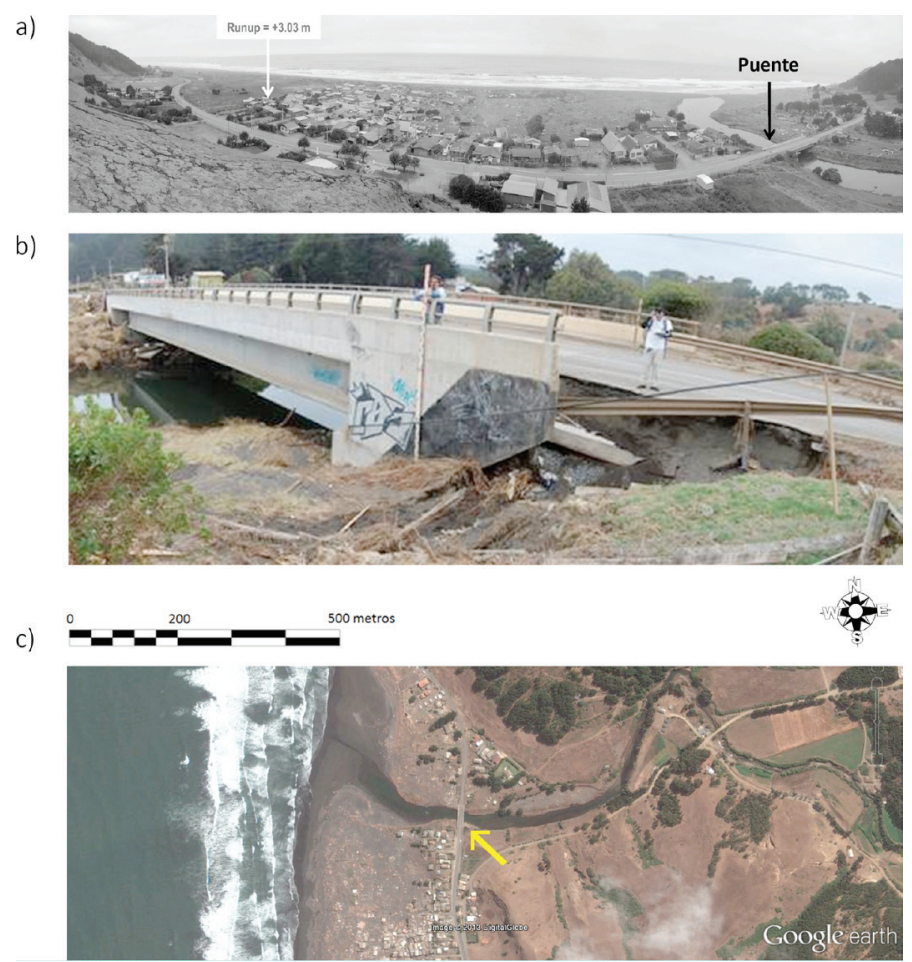

Figura 8: Falla por socavación del estribo del Puente Iloca $\left(34^{\circ} 56^{\prime}\right.$ S $)$, a) vista general de Iloca Sur, b) socavación notoria en los estribos del Puente Iloca y c) localización indicada con flecha

Las corrientes generadas por un tsunami pueden alcanzar velocidades importantes (Levin y Nosov, 2009) y como consecuencia provocar la socavación de instalaciones portuarias y costeras. El MOP (2010) reportó, a través de su cartografía de síntesis de daños, un total de 16 puntos en las carreteras costeras que fallaron por socavación, la mayoría correspondientes a puentes en estuarios donde las ondas de tsunami remontaron algunas veces varios kilómetros (Fritz et al., 2011). A modo de ejemplo, la Figura 8 ilustra la socavación del estribo del puente Iloca, ubicado en las cercanías del perfil de la Figura 4e. La Figura 9 muestra el fallo de la carpeta de rodado del camino costero en la ribera norte del Río Huenchollami.

a)

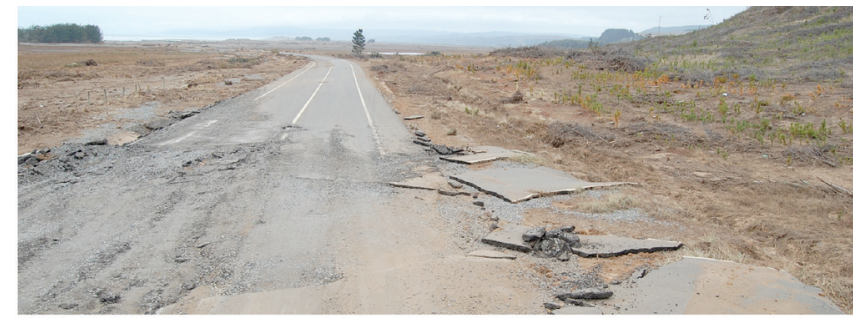

b)

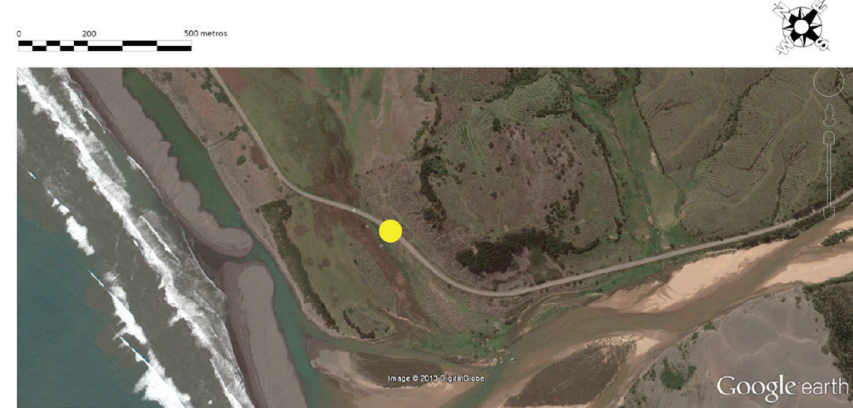

Figura 9: a) Falla en el camino costero ubicado en la ribera norte del Río Huenchollami (358'S), b) emplazamiento de la falla en el camino mostrada en a), distante a $500 \mathrm{~m}$ de la línea de costa en las inmediaciones de la desembocadura del Río Huenchollami.

Tabla 3: Número de infraestructuras costeras de uso público afectadas por el tsunami y organizadas por región.

\begin{tabular}{|l|c|c|c|c|c|c|c|}
\cline { 2 - 7 } \multicolumn{1}{c|}{} & \multicolumn{7}{c|}{ Región } \\
\hline $\begin{array}{l}\text { Tipo de } \\
\text { instalación }\end{array}$ & V & VI & VII & VIII & IX & XIV & Total \\
\hline $\begin{array}{l}\text { Muelle / sitio de } \\
\text { atraque }\end{array}$ & 4 & 0 & 1 & 2 & 0 & 0 & 7 \\
\hline $\begin{array}{l}\text { Instalaciones } \\
\text { portuarias y } \\
\text { pesqueras }\end{array}$ & 1 & 4 & 5 & 16 & 1 & 1 & 28 \\
\hline $\begin{array}{l}\text { Defensas } \\
\text { costeras }\end{array}$ & 0 & 0 & 1 & 0 & 1 & 2 & 5 \\
\hline $\begin{array}{l}\text { Accesos } \\
\text { portuarios }\end{array}$ & 0 & 0 & 0 & 1 & 0 & 0 & 1 \\
\hline TOTAL & 5 & 4 & 7 & 19 & 2 & 3 & 41 \\
\hline
\end{tabular}

Fuente: Adaptado de Gobierno (2010). 


\section{Pérdidas en embarcaciones menores}

El sector de los pescadores artesanales fue uno de los más afectados por este evento, principalmente por el daño a sus embarcaciones y la pérdida de motores, aparejos y artes de pesca. Se observaron desplazamientos significativos de embarcaciones menores en prácticamente toda la costa afectada, de pesqueros de mayor tonelaje en puertos como Talcahuano (36 43 'S) y de naves de gran tonelaje como el buque oceanográfico "Cabo de Hornos" en los Astilleros ASMAR Talcahuano. También se registraron desplazamientos de contenedores que, sumados a otros elementos flotantes, generaron daños en la infraestructura costera.

Un caso ilustrativo se encuentra en Dichato, a unos $50 \mathrm{~km}$ del epicentro. El buque de investigación Kay-Kay, una lancha con $20 \mathrm{~m}$ de eslora, fue arrastrada por el tsunami (Harris, 2010) más de $5 \mathrm{~km}$ desde su posición de anclaje original dentro de la bahía Coliumo, y depositada $750 \mathrm{~m}$ tierra adentro en la cabeza de la bahía (Hormazábal, C., 2010, com. pers.).

Más de 40 embarcaciones de pescadores artesanales resultaron dañadas en el puerto de San Antonio, con la pérdida de sus motores y todos los aparejos (IMSA, 2010). Lo mismo se observó en las más de 100 caletas de pescadores afectadas, que significó se perdiera un estimado del 55\% de la capacidad de esfuerzo de pesca de este sector (Marin et al., 2010).

La estadística de SERNAPESCA indica que entre los años 2010 y 2011 se registró una reducción del 19.7\% de embarcaciones inscritas por pescadores artesanales en cuatro regiones (Figura 10b). Ello equivale a 3070 botes con o sin motor y lanchas menores. Marin et al. (2010) estiman la pérdida en 1273 embarcaciones, pero su trabajo se restringe a tres regiones y no considera la pérdida de embarcaciones inscritas en regiones diferentes, situación común entre las embarcaciones inscritas en la región de Valparaíso. Desde el año 2004, es la primera oportunidad en que se reduce el número de embarcaciones, las que tenían una tendencia a incrementarse linealmente año a año.

La pérdida de embarcaciones puede explicar, en parte, el $17 \%$ de reducción en las toneladas de desembarque en 2010 respecto a 2009 (Figura 10a). Este descenso en 400000 toneladas en 2010 respecto a 2009 no es tan abrupto como el presentado en 2007 respecto a 2006, y que se explica debido al fenómeno El Niño (Zambrano, 2007).

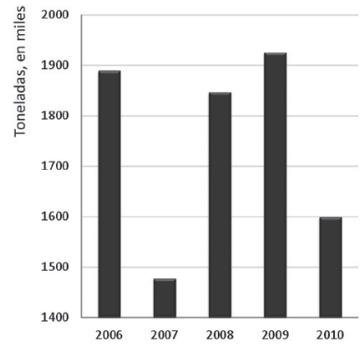

a)

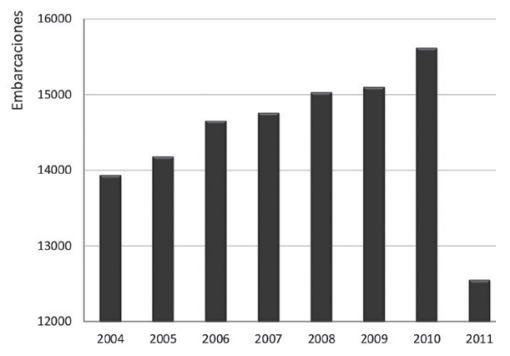

b)
Figura 10: a) Toneladas de desembarque de la pesca artesanal entre los años 2006 a 2010, se aprecia el descenso en 400000 toneladas del año 2010 respecto al año 2009 y b) número total de embarcaciones registradas por SERNAPESCA entre los años 2004 y 2011, se aprecia la pérdida de cerca de 3000 embarcaciones el año 2011. Las embarcaciones se registran a comienzo de año, mientras que los desembarques son contabilizados al final del año, de esta forma las embarcaciones del año 2011 corresponden al esfuerzo que actuó para los desembarques del año 2010. Fuente: SERNAPESCA (2012).

Tabla 4: Número de embarcaciones de pescadores artesanales inscritas entre la V y VIII región. En el resto de las regiones no se reportan pérdidas de embarcaciones. Fuente: SERNAPESCA (2012).

\begin{tabular}{|l|c|c|c|c|c|c|c|c|c|}
\hline $\begin{array}{c}\text { Tipo } \\
\text { embar- } \\
\text { cación }\end{array}$ & 2004 & 2005 & 2006 & 2007 & 2008 & 2009 & 2010 & 2011 & $\begin{array}{c}\% \\
\text { pérdida }\end{array}$ \\
\hline $\begin{array}{l}\text { Botes } \\
\text { remo o } \\
\text { vela }\end{array}$ & 623 & 667 & 707 & 727 & 766 & 784 & 772 & 528 & 31.6 \\
\hline $\begin{array}{l}\text { Bote } \\
\text { motor }\end{array}$ & 2497 & 2553 & 2578 & 2621 & 2725 & 2723 & 2910 & 2763 & 5.1 \\
\hline $\begin{array}{l}\text { Lancha } \\
\text { menor }\end{array}$ & 205 & 202 & 218 & 225 & 236 & 246 & 257 & 254 & 1.2 \\
\hline $\begin{array}{l}\text { Lancha } \\
\text { mediana }\end{array}$ & 171 & 175 & 180 & 181 & 176 & 183 & 185 & 182 & 1.6 \\
\hline $\begin{array}{l}\text { Lancha } \\
\text { mayor }\end{array}$ & 434 & 436 & 439 & 440 & 437 & 432 & 446 & 439 & 1.6 \\
\hline
\end{tabular}

\section{Conclusiones}

La zona costera de Chile se encuentra entre los lugares de mayor actividad sísmica del mundo. En promedio en los últimos cinco siglos, un terremoto destructivo de magnitud superior a 8 , se ha producido cada 10 años en alguna parte del territorio (Madariaga, 1998), y la mayoría de los distintos segmentos de la costa han sido el lugar de por lo menos uno de magnitud 8 durante los últimos 130 años. En el año 2010 sólo dos segmentos principales de la costa no habían presentado un terremoto $M_{w}=$ 8.0 por un tiempo prolongado, constituyendo lo que se denomina un gap sísmico maduro: Arica-Antofagasta y Constitución-Concepción. Esto motivó a que Ruegg et al. (2009) pronosticaran, sobre la base de mediciones 
de desplazamiento de la corteza, un megaterremoto de magnitud 8.0 a 8.5 para la zona afectada (Madariaga et al., 2010; Barrientos, 2010), trabajo que no era suficientemente conocido por las autoridades responsables para dar respuesta a este tipo de desastres naturales (Bitar, 2010; Fariña et al., 2012). Esto reveló la poca interacción existente entre científicos y las autoridades que deben dar respuesta a este tipo de sucesos en un país de gran actividad sísmica.

Al revisar los daños en infraestructura costera, se constata que el tsunami afectó a seis regiones del país. Fue responsable de la pérdida de 181 vidas humanas, equivalentes a un tercio de las ocasionadas por el terremoto y tsunami en su conjunto. Las tres cuartas partes de las víctimas del tsunami fueron niños y adultos mayores. Se constata una correlación positiva $\left(r^{2}=0.64\right)$ entre el runup y el porcentaje de víctimas en las localidades afectadas. Se registraron daños por socavación producto del ataque de las ondas de tsunami en al menos 16 puntos en las carreteras costeras. Cerca de 17000 viviendas fueron dañadas por el ingreso del mar, lo que corresponde al 8.5\% de la destrucción de viviendas provocada por el terremoto y 41 estructuras costeras mayores resultaron destruidas. Aproximadamente 3000 embarcaciones experimentaron daños -cerca del 20\% del total de embarcaciones inscritassiendo los pescadores artesanales los más afectados al perder el $31.6 \%$ de sus botes a remo o vela. El impacto en la industria pesquera consistió en una reducción del 17\% de las toneladas de desembarque con respecto al año anterior, disminución que no obstante fue menor a la experimentada el año 2007 producto del fenómeno El Niño.

\section{Agradecimientos}

La Dirección General de Investigación de la Universidad de Playa Ancha financió parcialmente este trabajo a través de los fondos DGI TES/01-2010 y DGI ING/03 2011. Colaboraron en la recolección de datos de campo: Alejandra Rojas, Ignacio Sepúlveda, Luis Burgos, Rodolfo Navarro, Matías Alday, Francisca Sánchez, Karla Sepúlveda, Luis Urbina, Ángel Lazcano, Esteban Retamal, Alejandra Rubio y Bozika Basic. El Sr. César Hormazábal proporcionó antecedentes importantes sobre los efectos del tsunami en Bahía Coliumo. El Sr. Gonzalo Bustillos colaboró en la extracción de datos poblacionales. Los revisores anónimos mejoraron sustancialmente el manuscrito con sus observaciones.

\section{Referencias}

Aránguiz, R. (2010). Modelación numérica del tsunami 2010 en la Bahía de Concepción utilizando deformación inicial estática no uniforme, Obras y Proyectos 8, 12 - 18

Astroza, A., Moroni, O., Brzev, S. and Tanner, J. (2012). Seismic assessment of engineered masonry buildings in the 2010 Maule Earthquake. Earthquake Spectra 28, No. S1, S385-S406

Barrientos, S. (2010). Terremoto $(\mathrm{m}=8.8)$ del 27 de febrero de 2010 en Chile. Revista de la Asociación Geológica Argentina 67(3): $412-420$

Benedetti, S. (2011). El terremoto más grande de la historia. Valdivia-Chile, 22 de mayo de 1960. Origo Ediciones, Santiago

Betanzo, R. (2010). Daños estructurales y lecciones del terremoto del 27/F en el Gran Concepción. Obras y Proyectos 8, 59 - 75

Bitar, S. (2010). Doce lecciones del terremoto chileno. Estado, Gobierno, Gestión Pública. Revista Chilena de Administración Pública 15-16: 7 - 18

Bresciani, L. (2010). Chile 27F 2010: La catástrofe de la falta de planificación. EURE. Volumen 36, Número 108: 151 - 153

Bray, J., Rollins, K., Hutchinson, T., Verdugo, R., Ledezma, C., Mylonakis, G., Assimaki, D., Montalva, G., Arduino, P., Olson, S.M., Kayen, R., Hashash, Y. and Candia, G. (2012). Effects of Ground Failure on Buildings, Ports, and Industrial Facilities. Earthquake Spectra 28, No. S1, S97-S118

Brunet, S., de la Llera, J. C., Jacobsen, A., Miranda, E. and Meza, C. (2012). Performance of Port Facilities in Southern Chile during the 27 February 2010 Maule Earthquake. Earthquake Spectra 28, No. S1, S513-S532

CEPAL (2010). The Chilean earthquake of 27 February 2010: an overview. United Nations publication. 2010-191, Santiago

Cereceda, P., Errázuriz, A.M. y Lagos, M. (2011). Terremotos y Tsunamis en Chile. Origo Ediciones, Santiago

Cisternas, M.E.(2011). Responsabilidad socialde los geocientistas en el manejo de riesgos: Enseñanzas derivadas de la experiencia chilena con el megaterremoto del 27 de febrero 2010. GOAL 2011: Geo-Risk Management a German-Latin American approach. Heidelberg, pp: 19 - 24, http://www.goaldaad. com.ar/goal/images/documentos/pubgoal2011.pdf (enero 2013) 
CONAPACH (2010). Diagnóstico de los impactos del terremoto y tsunami del 27 de febrero de 2010 en la pesca artesanal en Chile. Confederación Nacional de Pescadores Artesanales de Chile. http://www.conapach.cl/home/archivos/informe\%20final. doc (abril 2012).

Contreras, M., Winckler, P. y Urbina, L. (2012). Área de inundación y efectos del tsunami del 27 de febrero de 2010 en la localidad de Llolleo, San Antonio - Chile (3336.5"s). Revista Geográfica de Valparaíso, ํ 46: 69 - 81.

D'Ayala, D. and Benzoni, G. (2012). Historic and traditional structures during the 2010 Chile Earthquake: Observations, codes, and conservation strategies. Earthquake Spectra 28, No. S1, S425-S451

EERI (2010). The Mw 8.8. Chile Earthquake of February 27, 2010. Special Earthquake Report. http://www.eeri.org/site/images/eeri newsletter/2010_pdf/Chile10_insert.pdf (abril de 2013)

EM-DAT (2012). Emergency Events Database. Centre for Research on the Epidemiology of Disasters (CRED), School of Public Health of the Université Catholique de Louvain. http:// www.emdat.be/ (mayo de 2013)

Fariña, J., Aragoneses, N., Armesto, S., Almar, R., Bertness, M. y Silliman, B. (2010). Cambios en la estructura de hábitat de la Laguna Albufera, Humedal El Yali (V Región), luego del tsunami del 27 de Febrero del 2010. Boletín 01 Red Universitaria Cruz del Sur, Capítulo Ciencias del Mar. http://copas.udec.cl/ eng/news/Boletin_RUCS_01.pdf (febrero 2012).

Fariña, L.M., Opaso, C. y Vera-Puz, P. (2012). Impactos ambientales del terremoto y tsunami en Chile. Las réplicas ocultas del 27F. Fundación Terram, Santiago

Fiscalía (2011). Nomina de fallecidos y desaparecidos por el tsunami. Disponible en línea (fecha de visita febrero 2012): http://www. fiscaliadechile.cl/Fiscalia/sala_prensa/noticias_det.do?id=125

Fritz, H., Petroff, C., Catalán, P., Cienfuegos, R., Winckler, P., Kalligeris, N., Weiss, R., Barrientos, S., Meneses, G., ValderasBermejo, C., Ebeling, C., Papadopoulos, A., Contreras, M., Almar, R., Dominguez, J. and Synolakis, C. (2011). Field Survey of the 27 February 2010 Chile Tsunami. Pure and Applied Geophysics 168: 1989-2010

Gobierno (2010). Programa de Reconstrucción Terremoto y Maremoto del 27 de febrero de 2010, Resumen Ejecutivo. Ministerio del Interior, Ministerio de Obras Públicas, Ministerio de Hacienda, Ministerio Secretario General de la Presidencia,
Concepción. http://www.ministeriodesarrollosocial.gob.cl/pdf/ e60b893eb66a10139bfe68d2c6005636.pdf (Abril 2012)

González, P., Ortiz, J., Jerez,. R., Pavez, M. y Arcos, D. (2012). Efectos del tsunami 2010 en el humedal del río Mataquito. En Fariña, J.M. y Camaño, A. (editores) "Humedales costeros de Chile”. Ediciones UC, Santiago de Chile: 351 - 389

GORE (2010). Plan Reconstrucción Borde Costero PRBC18. Gobierno Regional del Bio-Bio. Disponible en línea: http:// www.minvu.cl/aopensite_20101207193158.aspx (visita en enero de 2013). Gobierno Regional del Bío Bío.

GEER (2010). Geo-engineering reconnaissance of the 2010 Maule, Chile Earthquake. Geo-Engineering Extreme Events Reconnaissance Association. Report No. GEER-022.

Harris, R. (2010). University of Concepcion Marine Station, Dichato, Chile. Journal of Plankton Research 32 (8): 1103 - 1104 HCDCh (2011a). Informe de la comisión especial investigadora del estado de la institucionalidad en relación a su capacidad de respuesta frente a desastres naturales. Honorable Cámara de Diputados de Chile. http://www.camara.cl/pdf.aspx?prmID=30 7\&prmTIPO=INVESTIGAFIN (visita julio 2012)

HCDCh (2011b). Informe de la comisión especial investigadora de procesos de adquisición, localización, distribución y asignación de viviendas de emergencia a damnificados por $27 \mathrm{~F}$. Honorable Cámara de Diputados de Chile. http://www.camara. $\mathrm{cl} /$ pdf.aspx?prmID=529\&prmTIPO=INVESTIGAFIN

Herd, D.G., Youd, T.L., Meyer, H., Arango, J.L., Person, W.J. and Mendoza, C. (1981). The Great Tumaco, Colombia Earthquake of 12 December 1979. Science 211, N4481: 441 - 445

IMSA (2010). Acta Concejo Municipal San Antonio, sesión ordinaria $n^{\circ} 07$ (3 de marzo de 2010). Ilustre Municipalidad de San Antonio. (última visita febrero 2012) http://transparencia. sanantonio.cl/Lists/Actas\%20de\%20Concejo/Attachments/55/ ActaSONo07_03-02-2010.pdf.

INE (2010). Información cartográfica efectos tsunami 2010. Instituto Nacional de Estadísticas. Disponible en línea: http://www.ine.cl/canales/sala_prensa/noticias/2010/marzo/ cartografia.php (enero de 2013).

INE (2013). Población total por entidades poblacionales. Censo Población y Vivienda 2002. Sistema Recuperación datos áreas medianas y pequeñas Redatam+-CELADE-CEPAl, ONU. Instituto Nacional de Estadísticas. Disponible en CD. 
INN (2003). Análisis y Diseño de Edificios con Aislación Sísmica, NCh 2745 Of. 2003. Instituto Nacional de Normalización, Santiago, Chile

INN (2003a). Diseño Sísmico de Estructuras e Instalaciones Industriales, NCh 2369 Of. 2003. Instituto Nacional de Normalización, 120pp.

INN (2009). Diseño sísmico de edificios, NCh 433 Of 96, modificada 2009. Instituto Nacional de Normalización

Jaramillo, E., Manzano, M., Morales, G. y Velásquez, C. (2012). Interacción entre defensas costeras artificiales en playas arenosas y perturbaciones naturales de gran escala: el terremoto del $27 \mathrm{~F}$ de 2010 en la costa del centro-sur de Chile. Revista de Geografía Norte Grande 53: 75 - 84

Kanamori, H. (1978). Quantification of Earthquakes. Nature 271, No 5644: $411-414$

La Tercera (2011). ¿Cuánto le costó el terremoto a Chile?. Cuerpo de Reportajes domingo 20 de febrero 2011. Periodista: David Lefin L. http://diario.latercera.com/2011/02/20/01/contenido/ reportajes/25-59879-9-cuanto-le-costo-el-terremoto-a-chile. shtml (mayo de 2013)

Larrañaga, O. y Herrera, R. (2010). Encuesta Post Terremoto 2010: Principales resultados. Efectos en la calidad de vida de la población afectada por el terremoto/tsunami. Ministerio de Planificación y PNUD, Santiago.http://www.redatam.org/chl/ ENCPT/documentos/informe_encuesta_post_terremoto.pdf (última visita mayo 2013)

Lazcano, A. y Retamal, E. (2011). Diagnóstico ambiental en la zona costera del sector Llolleo, comuna de San Antonio, Chile, por efectos revelados del tsunami 2010. Tesis de Ingeniero Ambiental, Universidad de Playa Ancha, Valparaíso

Levin, B. and Nosov, M. (2009). Physics of Tsunami. Springer

Madariaga, R. (1998). Sismicidad de Chile. Física de la Tierra 10: $221-258$

Madariaga, R., Métois, M., Vigny, C. and Campos, J. (2010). Central Chile Finally Breaks. Science 328: 181 - 182

Martínez, C., Rojas, O., Jaque, E., Quezada, J., Vázquez, D. y Belmonte, A. (2011). Efectos territoriales del tsunami del 27 de febrero de 2010 en la costa de la región del Bio-bío, Chile. Revista Geográfica de América Central 47 E(2), 1 - 16
Marín, A., Gelcich, .S., Araya, G., Olea, G., Espíndola, M. and Castilla, J.C. (2010). The 2010 tsunami in Chile: Devastation and survival of coastal small-scale fishing communities. Marine Policy 34: 1381 - 1384

MINVU (2010). Plan de Reconstrucción MINVU “Chile Unido Reconstruye Mejor". Ministerio de Vivienda y Urbanismo, Gobierno de Chile, 3era edición

MOP (2010). Carta síntesis daño de infraestructura terremoto / tsunami 2010. Ministerio de Obras Públicas, disponible en línea (fecha última visita en abril de 2013): http://www.snit.cl/ terremoto2010/mapas_nacionales/mop/Carta_Sintesis_Danos_ Infraestructura_MOP_12_03_2010.rar

Morales, R. (2010). Terremoto y tsunami del 27 de febrero de 2010. Efectos urbanos en localidades de la provincia de Arauco. Urbano 22: 43 - 62

Morton, R.A., Buckley, M.L., Gelfenbaum, G., Richmond, B.M., Cecioni, A., Artal, O., Hoffman, C., and Perez, F. (2010). Geological impacts and sedimentary record of the February 27, 2010, Chile tsunami; La Trinchera to Concepcion: U.S. Geological Survey Open-File Report 2010-1116, 22pp. Disponible en línea: http://pubs.usgs.gov/of/2010/1116/ (abril de 2013).

Music, J., Tapia, P. y Fleming, W. (2012). Implicancia del Decreto 117 en la demanda sísmica y respuesta global en edificios de Antofagasta. Obras y Proyectos 11, 19-26

Nahuelpan, E. y Varas, J. (2010). El Terremoto/Tsumami en Chile. Una mirada a las estadísticas médico legales.

Olsen, M.J., Cheung, K.F., Yamazaki, Y., Butcher, S., Garlock, M., Yim, S., McGarity, S., Robertson, I., Burgos, L. and Young Y.L. (2012). Damage Assessment of the 2010 Chile Earthquake and Tsunami Using Terrestrial Laser Scanning. Earthquake Spectra 28, No. S1, S179-S197

OPS (2010). El Terremoto y Tsunami del 27 de Febrero en Chile: Crónica y lecciones aprendidas en el sector salud. Organización Panamericana de la Salud. Santiago

Pulido, N., Sekiguchi, T., Shoji, G., Alba, J., Lazares, F. and Saito T. (2011). Earthquake Source Process and Site Effects of Strong Motion Stations of the 2010 Chile Mega-Earthquake. 2010 Chile Earthquake and Tsunami Technical Report, JSTJICA SATREPS TEAM, 1-9. http://ares.tu.chiba-u.jp/peru/pdf/ output/tr/PulidoJST_reportI_1.pdf (abril de 2013) 
Quezada, J., Jaque, E., Belmonte, A., Fernández, A., Vásquez, D., y Martínez, C. (2010). Movimientos cosísmicos verticales y cambios geomorfológicos generados durante el terremoto $\mathrm{Mw}=$ 8,8 del 27 de febrero de 2010 en el centro - sur de Chile. Revista Geográfica del Sur 2: $11-45$

Quezada, J., Jaque, E., Belmonte, A., Fernández, A., Vásquez, D., y Martínez, C. (2010a). Cambios geomorfológicos por el evento sísmico del 27 de febrero 2010 en el centro - sur de Chile. Obras y Proyectos 8: 4 - 11

Quezada, J., Jaque, E., Fernández, A. y Vásquez, D. (2012). Cambios en el relieve generados como consecuencia del terremoto $\mathrm{Mw}=8,8$ del 27 de febrero de 2010 en el centro-sur de Chile. Revista de Geografía del Norte Grande 53: 35 - 55

Robertson, I., Chock, G. and Morlab, J. (2012) Structural Analysis of Selected Failures Caused by the 27 February 2010 Chile Tsunami. Earthquake Spectra 28, No. S1, S215-S243.

Romero, H., Fuentes, C. and Smith, P. (2010). Dimensiones geográficas territoriales, institucionales y sociales del terremoto de Chile del 27 de febrero de 2010. Cuadernos de Geografia: Revista Colombiana de Geografía 19: 135-150

Rubio, A. y Basic, B. (2011). Investigación de impactos ambientales post tsunami Chile 2010 en la laguna albufera perteneciente al humedal El Yali (3345’30”S). Tesis de Ingeniero Ambiental, Universidad de Playa Ancha, Valparaíso, Chile

Ruegg, J., Rudloff, A., Vigny, C., Madariaga, R., de Chabaliera, J., Campos, J., Kausel, E., Barrientos, S. and Dimitrov, D. (2009). Interseismic strain accumulation measured by GPS in the seismic gap between Constitución and Concepción in Chile. Physics of the Earth and Planetary Interiors 175: 78-85

Saragoni, R. (2011). El mega terremoto del Maule de 2010: una lección de buena ingeniería, pero con sorpresas y nuevos desafíos. Revista Anales de la Universidad de Chile, Séptima Serie, $\mathrm{N}^{\circ} 1: 37$ - 56. http://www.sye.uchile.cl/index.php/ANUC/ article/viewFile/12247/18113 (abril 2012)

SERNAPESCA (2012). Anuarios Estadísticos. Servicio Nacional del Pesca. Disponible en línea (enero de 2013): http:// www.sernapesca.cl/index.php?option $=$ com_remository\&Itemid $=54 \&$ func $=$ select\&id $=2$

Synolakis, C and Okal, E. (2005). 1992-2002 perpective on a decade of post-tsunami surveys. In "Tsunamis: Case Studies and Recent Developments”, K. Satake (ed.), 1-29
UNESCO-IOC (1998). Post-Tsunami Survey Field Guide (First Edition). UNESCO-IOC. Intergovernmental Oceanographic Commission Manuals and Guides \# 37: http://ioc.unesco.org/ itsu/files/MG037.pdf (mayo 2013).

Urbina, L. (2011). Variación de los factores ambientales en las lagunas costeras de Llolleo, San Antonio - Chile, producto del tsunami del 27/02/2010. Tesis de Ingeniero Ambiental, Universidad de Playa Ancha, Valparaíso

Valdovinos, C., Sandoval, N., Vásquez, D. y Olmos, V. (2012). El humedal costero Tubul - Raqui: un ecosistema chileno de alto valor de conservación severamente perturbado por el terremoto del 2010. En Fariña, M.J. y Camaño, A. (editores) “Humedales costeros de Chile”. Ediciones UC, Santiago de Chile: 391 - 437

Vargas, G., Farías, M., Carretier, S., Tassara, A., Baize, S. and Melnick, D. (2011). Coastal uplift and tsunami effects associated to the 2010 Mw8.8 Maule earthquake in Central Chile. Andean Geology 38 (1): 219-238

Vigny, C., Socquet, A., Peyrat, S., Ruegg, J.C., Métois, M., Madariaga, R., Morvan, S., Lancieri, M., Lacassin, R., Campos, J., Carrizo, D., Bejar-Pizarro, M., Barrientos, S., Armijo, R., Aranda, C., Valderas-Bermejo, M.C., Ortega, I., Bondoux, F., Baize, S., Lyon-Caen, H., Pavez, A., Vilotte, J.P., Bevis, M., Brooks, B., Smalley, R., Parra, H., Baez, J.C., Blanco, M., Cimbaro, S. and Kendrick, E. (2011). The 2010 Mw 8.8 Maule Megathrust Earthquake of Central Chile, Monitored by GPS. Science 332: 1417 - 1421

Westenenk, B., de la Llera, J.C., Besa, J.J., Jünemann, R., Jack Moehle, J., Lüders, C., Inaudi, J.A., Elwood, K and Hwang, S.J. (2012). Response of Reinforced Concrete Buildings in Concepción during the Maule Earthquake. Earthquake Spectra 28, No. S1, S257-S280.

Winckler, P., Reyes, M., Sepúlveda, I. y Molina, M. (2010). Observaciones de campo del tsunami del 27 de febrero de 2010 en Isla Robinson Crusoe, Archipiélago Juan Fernández. Revista de la Sociedad Chilena de Ingeniería Hidráulica 25(1): 12pp.

Winckler, P., Reyes, M. y Contreras, M. (2011). Recomendaciones de diseño de obras marítimas y terrestres sometidas a cargas de tsunami. Anales del Instituto de Ingenieros de Chile, 462: 19-39.

Yasuda, S., Verdugo, R., Konagai, K., Sugano, T., Villalobos, F., Okamura, M., Tobita, T., Torres, A. and Towhata, I. (2010). Geotechnical damaged caused by the 2010 Maule, Chile earthquake. ISSMGE Bulletin 4(2), 16-27 
Zambrano, E. (2007). El Niño 2006-2007. Desarrollo y manifestaciones en aguas ecuatorianas. Acta Oceanográfica del Pacífico 14(1): 1 - 6

Zareian, F., Aguirre, C., Beltrán. J.F., Cruz, E., Herrera, R., Leon, R., Millan, A. and Verdugo, A. (2012). Reconnaissance Report of Chilean Industrial Facilities Affected by the 2010 Chile Offshore Bío-Bío Earthquake. Earthquake Spectra 28, No. S1, S513-S532 